\title{
Measurement of Atom Resolvability in CryoEM Maps with Q-scores
}

Greg Pintilie

\section{Stanford U., Stanford, California, United States}

CryoEM maps are now at the point where resolvability of individual atoms can be achieved. However, resolvability is not necessarily uniform throughout the map. We introduce a quantitative parameter to characterize the resolvability of individual atoms in cryoEM maps, the map Q-score. Q-scores can be calculated for atoms in proteins, nucleic acids, water, ligands, and other solvent atoms, using models fitted to or derived from cryoEM maps. Averaged over entire models, Q-scores correlate very well with the estimated resolution of cryoEM maps for both protein and RNA. Assuming the models they are calculated from are well-fitted to the map, Q-scores can be used as a measure of resolvability in cryoEM maps at various scales, from entire macromolecules down to individual atoms. Q-score analysis of multiple cryoEM maps of the same proteins derived from different labs confirms the reproducibility of structural features from side chains down to water and ion atoms.

We tested the effect of several factors on Q-scores. First, using the cross-correlation about the mean makes the Q-scores insensitive to the height and vertical offset of the reference Gaussian. This means that as long as map values are decreasing around an atom, regardless of their relative magnitude in the map, the Qscore for the atom could still be high. Second, small changes in grid step and placement do not affect the Q-score; however if the grid step is too large relative to the resolution of the map, resolvability and also Q-scores can start to decrease. Finally, sharpening can increase the visible detail in the map along with Qscores, but Q-scores start to decrease if excessive sharpening is applied.

Q-scores measure the resolvability of individual atoms in a cryoEM map, using an atomic model fitted to or built into the map. It should be noted that nothing is assumed about the model itself, e.g. whether it has good stereochemistry; this could be deduced with other scores such as the Molprobity score ${ }^{31}$. Q-scores averaged over entire models correlate very closely to the reported resolution of the maps in which they are calculated. The score can also be useful to analyze the map and its resolvability in different regions, and also test whether the model may need further refinement in some areas as indicated by low Q-scores. Here, Q-scores were also applied to various maps at different resolutions to show quantifiable trends across different side chains in proteins, bases in nucleic acids, and also to assess the resolvability of solvent atoms and ions. Q-scores should continue to be a useful metric in the analysis of cryoEM maps and models.

References

https://www.nature.com/articles/s41592-020-0731-1 